\title{
Perisaccadic Compression Correlates with Saccadic Peak Velocity: Differential Association of Eye Movement Dynamics with Perceptual Mislocalization Patterns
}

\author{
Florian Ostendorf, Constance Fischer, Carsten Finke, and Christoph J. Ploner \\ Klinik für Neurologie, Charité, 10117 Berlin, Germany
}

\begin{abstract}
Objects flashed around the onset of a saccadic eye movement are grossly mislocalized. Perisaccadic mislocalization has been related to a spatiotemporal misalignment of an extraretinal eye position signal with the corresponding saccade. Two phenomena have been observed: a systematic shift of perceived positions in saccade direction and an additional compression toward the saccade target. At present, it is unclear whether these two components of mislocalization are mediated by distinct mechanisms and how extraretinal signals may contribute to either of them. Moreover, the pattern and strength of perisaccadic mislocalization varies considerably across studies and even between subjects tested under identical conditions. Here, we investigated whether interindividual differences in saccade parameters are related to differences in mislocalization. We found that the individual strength of perceptual compression selectively correlates with the peak velocity of corresponding saccades. Other saccade parameters did not correlate with compression. No correlation was found between the shift component of perisaccadic mislocalization and any saccade parameter. This dissociation suggests that shift and compression components are, at least partially, mediated by distinct mechanisms. Because neuronal activity in the superior colliculus and downstream oculomotor areas has been shown to correlate with saccadic peak velocity, our findings support the notion that a reafferent extraretinal signal associated with saccadic motor commands may contribute to perisaccadic compression of perceived positions.
\end{abstract}

Key words: human; eye movement; saccade; extraretinal; space constancy; localization

\section{Introduction}

To explore a visual scene, we perform frequent saccadic eye movements. Although the retinal image changes across each saccade, the brain somehow integrates the rapid series of snapshots to a coherent and stable representation of the entire visual scene. It has been proposed that extraretinal eye position signals may provide the visual system with information on planned or ongoing changes of eye position to maintain space constancy across saccades (Sperry, 1950; von Holst and Mittelstaedt, 1950). Probing space constancy under laboratory conditions, however, revealed a systematic mislocalization of briefly flashed stimuli within a restricted time window around saccade onset. Two components of this perisaccadic mislocalization have been identified: first, a uniform shift of apparent positions in saccade direction, independent of the position of the flashed stimulus in the visual field (Matin and Pearce, 1965; Honda, 1989; Dassonville et al., 1995; Cai et al., 1997), and second, a localization bias ("compression") toward the saccade target, i.e., stimuli presented beyond the saccade target are mislocalized against saccade direction

Received March 10, 2007; revised May 24, 2007; accepted May 25, 2007.

This work was supported by German Federal Ministry of Education and Research Grant 01 GW0653 (Visuospatial(ognition). We thank two anonymous reviewers for helpful comments on a previous version of this manuscript.

Correspondence should be addressed to Florian Ostendorf, Klinik für Neurologie, Charité, Charitéplatz 1, D-10117 Berlin, Germany. E-mail: florian.ostendorf@charite.de.

DOI:10.1523/JNEUROSCI.2074-07.2007

Copyright $\odot 2007$ Society for Neuroscience $\quad$ 0270-6474/07/277559-05\$15.00/0
(Honda, 1993; Morrone et al., 1997; Ross et al., 1997; Lappe et al., 2000; Kaiser and Lappe, 2004).

The phenomenon of perisaccadic mislocalization may give insight into cerebral mechanisms of transsaccadic perceptual stability. Perisaccadic mislocalization has been assumed to reflect the spatiotemporal dynamics of an extraretinal eye position signal that is not perfectly aligned with the corresponding saccade (Ross et al., 2001; Schlag and Schlag-Rey, 2002). Alternatively, visual factors such as afferent delays, visual persistence of the flashed stimulus, and masking by retinal image blur could also account for perceptual mislocalization before fast retinal image motion (Schlag and Schlag-Rey, 2002; Pola, 2004; Ostendorf et al., 2006). The specific spatiotemporal dynamics and respective contributions of shift and compression components to perisaccadic mislocalization vary considerably across studies and render comparisons difficult. Stimulus-associated factors such as the perisaccadic availability of visual references, the contrast, and eccentricity of the flashed stimulus have been shown to modify the pattern of perisaccadic mislocalization and may explain part of this variability (Honda, 1993; Dassonville et al., 1995; Lappe et al., 2000; Kaiser and Lappe, 2004; Michels and Lappe, 2004). To date, it therefore remains a matter of debate how visual and extraretinal factors contribute to transsaccadic integration. In addition, there appears to be considerable variability in mislocalization patterns between subjects, even when tested with identical stimuli (Honda, 1993; Morrone et al., 1997; Ostendorf et al., 
2006). Here, we used this interindividual variability to investigate the contribution of extraretinal signals to mislocalization. We reasoned that, if perisaccadic mislocalization is indeed related to an extraretinal signal associated with the saccadic motor command, the individual spatiotemporal dynamics of perisaccadic mislocalization should exhibit a relation to parameters of the corresponding saccade.

\section{Materials and Methods}

Subjects. Ten male subjects (20-29 years of age) participated in the experiment. All subjects had normal vision. They were naive as to the purpose of the experiment and were paid for participation. Informed consent was obtained from all subjects before participation in the study, which was approved by the local ethics committee and conducted in conformity with the Declaration of Helsinki.

Stimuli and experimental setup. Subjects sat in front of a 22 inch monitor, subtending $43.6 \times 33.4^{\circ}$ of visual angle at a distance of $50 \mathrm{~cm}$, with their head fixed to the recording system by means of a chinrest and a bite bar. Experiments were performed in a darkened room. Stimuli were generated at a frame rate of $110 \mathrm{~Hz}$ by a visual stimulus generator (Experimental Run Time System; BeriSoft, Frankfurt, Germany) housed in a personal computer.

Procedure. All stimuli were presented on a homogenous gray background (luminance, $3 \mathrm{~cd} / \mathrm{m}^{2}$ ). Each trial started with the presentation of a green fixation $\operatorname{dot}\left(0.4^{\circ}\right.$ in diameter; luminance, $\left.13 \mathrm{~cd} / \mathrm{m}^{2}\right) 5^{\circ}$ right or left of screen center. Subjects were asked to perform a saccade from this initial fixation point to a newly appearing stimulus with the same size and luminance at the mirror-image position of the fixation point, either $5^{\circ}$ left or right of screen center (Fig. 1). Saccade direction was fixed in a block of trials. Both the fixation point and saccade target point remained illuminated until the end of a trial. At an unpredictable interval after target onset $(100-350 \mathrm{~ms})$, a vertical bar $\left(0.7 \times 33.4^{\circ}\right.$; luminance, 13 $\mathrm{cd} / \mathrm{m}^{2}$ ) was flashed at one of three possible screen positions for one video frame $(9 \mathrm{~ms})$. This interstimulus interval corresponded to an average presentation time of $150 \mathrm{~ms}$ before up to $100 \mathrm{~ms}$ after saccade onset for the flashed bar, given the average saccadic reaction time (SRT) in our study (mean \pm SD SRT, $250 \pm 29 \mathrm{~ms}$ ). Bar positions were located at screen center $\left(0^{\circ}\right)$ or $-10^{\circ}$ left or $10^{\circ}$ right of screen center. At $750 \mathrm{~ms}$ after saccade target onset, a horizontal reference ruler appeared together with a mouse pointer. The ruler extended over the whole screen and had small vertical tick lines at $1^{\circ}$ intervals. Subjects moved the mouse pointer to the apparent position of the flashed bar and pushed the left mouse button. During this experimental phase, subjects were free to move their eyes. At least 180 trials were collected per subject and saccade direction.

Data analysis. Horizontal position of the right eye was sampled at 200 $\mathrm{Hz}$ by horizontal infrared oculography (AMTech Eye Tracker; AMTech, Weinheim, Germany). Calibration was performed regularly throughout the recording session. Data were analyzed off-line. Saccade onset was defined by using a velocity criterion (threshold, $35 \%$ ). Trials were discarded when no saccade occurred before ruler onset, the saccade end point fell outside a $4^{\circ}$ window around the saccade target point, a blink occurred around bar presentation, or a corrective saccade occurred before ruler onset (mean, $8 \%$ ). In trials that met task requirements, saccade parameters were analyzed. We measured saccadic peak velocity, saccadic reaction time, and mean amplitude. For further analysis, rightward and leftward saccades were pooled. Therefore, all positional information for trials with leftward saccades was inversed. Presentation time of the flashed vertical bar was determined with respect to saccade onset.

\section{Results}

Figure 2 shows individual responses of two representative subjects (Fig. 2A,B), aligned on saccade onset. Shown are the perceived positions of the flashed bar for real bar positions at $-10^{\circ}$ (black triangles), $0^{\circ}$ (gray circles), and $10^{\circ}$ (gray triangles) eccentricity. Responses of both subjects show a systematic mislocalization of the flashed bar around saccade onset with a shift of apparent positions in saccade direction for bars presented at $-10^{\circ}$ and
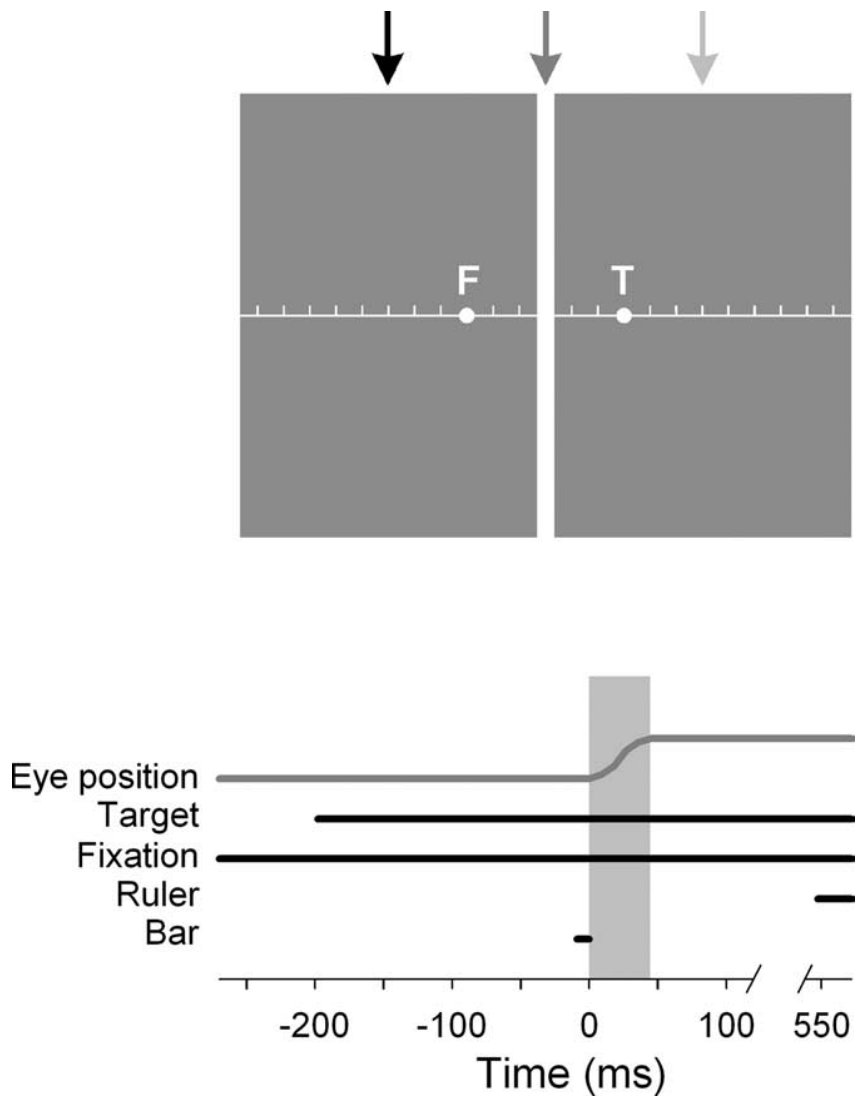

Figure 1. Top, Spatial layout of the screen. Screen background was gray and $40 \times 30 \mathrm{~cm}$ $\left(43.6 \times 33.4^{\circ}\right.$ at a distance of $\left.50 \mathrm{~cm}\right)$. An initial fixation point $(\mathrm{F})$ was presented at $5^{\circ}$ either right or left of screen center. A second stimulus, the saccade target $(\mathrm{T})$, was presented at the respective mirror location. A vertical bar was flashed at either screen center (as shown here) or $10^{\circ}$ right or $-10^{\circ}$ left of screen center, respectively (arrows mark possible locations). After saccade execution, a ruler was presented across the screen together with a mouse pointer. Bottom, Time course of experimental setup. Subjects fixated the fixation point. At a variable time, the saccade target was presented; both stimuli remained illuminated until the end of a trial. Subjects performed a saccade (gray shaded area) from the fixation point to the target stimulus. At a variable time around saccade onset, the vertical bar was flashed for $9 \mathrm{~ms}$. A ruler was switched on $750 \mathrm{~ms}$ after target onset.

$0^{\circ}$. In contrast, at position $10^{\circ}$, a systematic mislocalization against saccade direction evolved around saccade onset for subject FB (Fig. 2 A), implying a compression of perceived positions toward the saccade target position $\left(5^{\circ}\right)$. This pattern of perisaccadic mislocalization resembles the findings of previous reports (Honda, 1993; Morrone et al., 1997; Lappe et al., 2000). However, the pattern of mislocalization clearly differs between both subjects, with subject FB (Fig. $2 A$ ) showing a strong and subject RW (Fig. 2B) showing a rather weak compression around saccade onset.

To quantify the individual strength of shift and compression of apparent positions, we calculated two index measures (Lappe et al., 2000): The shift index is defined as the mean of the three apparent bar positions for a given point of time, normalized to their mean positions $100 \mathrm{~ms}$ before and after saccade onset. Compression is expressed as an index of apparent separation that is defined as mean separation between apparent bar positions normalized to mean separation between real bar positions for a given point of time. Thus, an index value of 1 indicates no compression and a value of 0 would indicate maximal compression with bars presented at all three positions seen on a single position.

Shift and apparent separation values for the two subjects are 
A

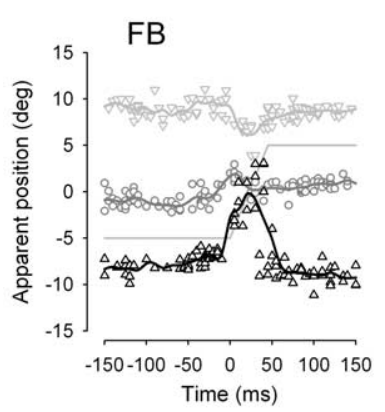

C
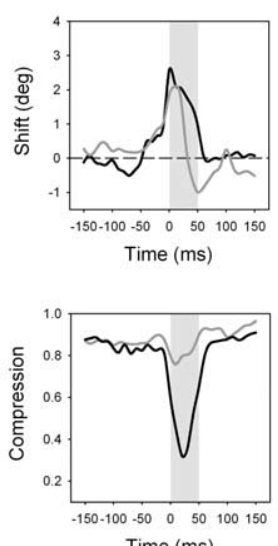

Time (ms)
B

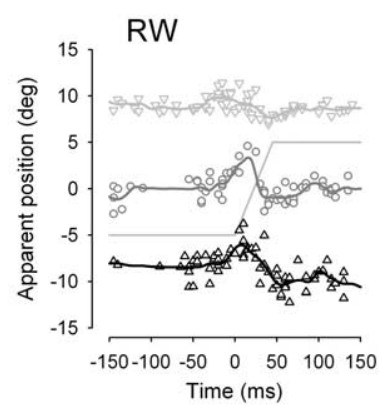

D

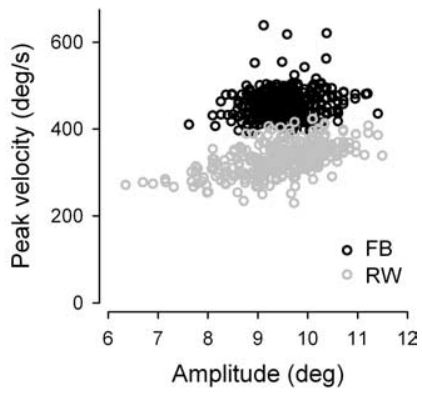

Figure 2. $\quad A, B$, Representative responses of a subject with strong (FB) and a subject with weak (RW) compression of apparent positions around saccade onset. Apparent positions of the flashed bar are aligned on saccade onset. The gray line indicates eye position. Symbols represent responses for real bar positions of $-10^{\circ}$ (black triangles), $0^{\circ}$ (gray circles), and $10^{\circ}$ (gray triangles), respectively. Continuous lines are weighted running averages through the data. C, Compression and shift of the three apparent bar positions for a given point of time with respect to saccade onset for the same two subjects. The shift is calculated as the average of the three apparent bar positions normalized to the average apparent bar positions $100 \mathrm{~ms}$ before and after saccade onset, respectively. Space compression is calculated as the mean separation between apparent bar positions normalized to mean separation between real bar positions. $\boldsymbol{D}$, Saccade peak velocities of the same two subjects, plotted against saccade amplitudes.

shown in Figure 2C. The shift of apparent positions in saccade direction peaks around saccade onset in both subjects and its maximum value is slightly higher for subject $\mathrm{FB}$ compared with subject RW (shift in saccade direction, $2.57^{\circ}$ and $2.1^{\circ}$, respectively). Compression peaks $10-20 \mathrm{~ms}$ after saccade onset and is much more pronounced in subject FB than in subject RW (minimum apparent separation, 0.32 and 0.76 , respectively). We next analyzed the parameters of corresponding saccades. Saccade peak velocities for subject FB (black dots) and subject RW (gray dots) are plotted against corresponding saccade amplitudes in Figure $2 D$, showing the typical main sequence relationship (Bahill et al., 1975 ). Both sets of saccades are hypometric on average (mean \pm SD amplitude, $9.53 \pm 0.56^{\circ}$ for subject FB and $9.46 \pm 0.84^{\circ}$ for subject RW, respectively). Furthermore, it is evident that saccades of subject FB show higher peak velocities with an upwards shifted main sequence relationship (mean \pm SD peak velocity, $456.9 \pm 33^{\circ} / \mathrm{s}$ for subject FB and $329.7 \pm 37^{\circ} \%$ for subject RW, respectively). Average SRTs were shorter for subject FB (mean \pm SD SRT, $229 \pm 55 \mathrm{~ms}$ for subject FB and $299 \pm 65 \mathrm{~ms}$ for subject RW, respectively).

To analyze a possible relationship between perisaccadic mislocalization and corresponding saccade parameters across all
A

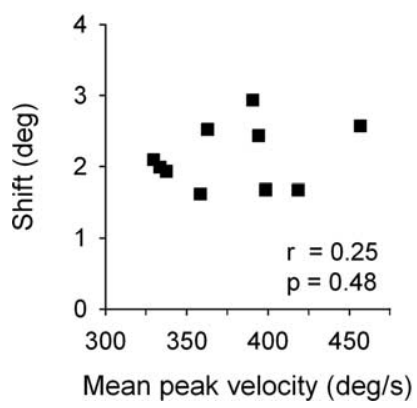

B

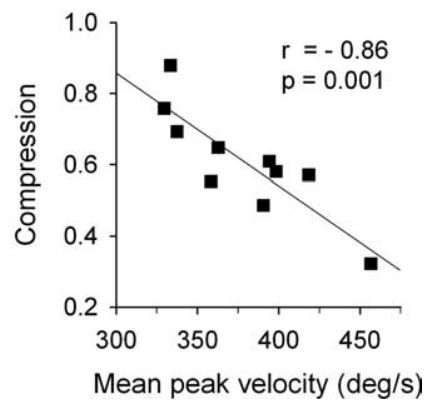

Figure 3. A, Shift of apparent bar positions versus mean saccadic peak velocity. $\boldsymbol{B}$, Perceptual compression expressed as minimum apparent bar separation versus mean saccadic peak velocity for the 10 subjects tested. Data were fitted with a linear regression line.

subjects, we analyzed the strength of both mislocalization components as a function of corresponding saccade parameters. No correlation was observed between the shift component of perisaccadic mislocalization and mean saccadic peak velocity (Fig. 3A) (Pearson's correlation coefficient, $r=0.25 ; p=0.48$ ), saccade amplitude $(r=0.12 ; p=0.75)$, or SRT $(r=0.09 ; p=$ 0.81 ). The compression component did not correlate with mean saccade amplitude $(r=-0.37 ; p=0.29)$ or SRT $(r=-0.05 ; p=$ $0.89)$. However, linear regression confirmed a highly significant correlation between individual compression strength and the mean peak velocity of corresponding saccades $(r=-0.86 ; p=$ 0.001 ), i.e., higher saccadic peak velocities were associated with a stronger perceptual compression around saccade onset. Figure $3 B$ shows maximum compression values as a function of mean saccadic peak velocity for the 10 subjects tested.

Differential visual stimulation induced by the variable displacement speed of the retinal image may explain at least part of the observed relationship between saccadic peak velocity and perceptual compression. Additional analyses, however, indicate that differences of a possible intrasaccadic spread of the flashed bar across the moving retina or varying spatiotemporal uncertainty of the flashed bar representation with different saccade velocities cannot explain the slope of the observed correlation (supplemental Figs. 1, 2, available at www.jneurosci.org as supplemental material).

\section{Discussion}

In the present study, we asked whether the spatiotemporal dynamics of perisaccadic mislocalization exhibit a relationship to the characteristics of corresponding saccadic eye movements. We found that the strength of the compression component varies with the peak velocity of corresponding saccades, whereas no correlation with saccadic reaction time or mean amplitude was observed. The shift component of perisaccadic mislocalization did not vary with any of the analyzed saccade parameters. In the following, we discuss the implications of this finding with respect to the possible differential contributions of visual and extraretinal signals to the shift and compression components of perisaccadic mislocalization.

Perisaccadic mislocalization has been assumed to reflect the dynamics of an extraretinal eye position signal (Ross et al., 2001; Schlag and Schlag-Rey, 2002). If this assumption holds true, one should expect some relation of perisaccadic mislocalization to the parameters of corresponding saccadic eye movements. Indeed, recent experimental work indicates that the compression component scales with saccade amplitude (Morrone et al., 1997; Kaiser 
and Lappe, 2004) and that perisaccadic compression is centered on the saccade end point in experimentally induced saccade adaptation (Awater et al., 2005). In our study, we did not observe a dependence of perisaccadic mislocalization on saccade amplitudes. This negative finding may be easily explained by the use of one fixed target eccentricity $\left(10^{\circ}\right)$ and the resulting small interindividual differences in mean saccade amplitudes in our study (mean amplitude range, 9.1-9.9 $9^{\circ}$. However, we found a strong dependence of perisaccadic perceptual compression on saccadic peak velocity. Our study, therefore, supports the notion that the actual parameters of corresponding saccades contribute to the specific spatiotemporal dynamics of perceptual mislocalization around saccadic eye movements (Morrone et al., 2005; Awater and Lappe, 2006).

The observation of a covariation of the compression but not the shift component with saccadic peak velocity supports the idea of partially dissociable mechanisms for both components of perceptual mislocalization. This is consistent with previous studies demonstrating a differential susceptibility of either component to specific experimental manipulations: the compression but not the shift component has been shown to vary with stimulus contrast (Michels and Lappe, 2004). Perisaccadic compression has been shown to depend on the availability of visual references after saccade execution (Lappe et al., 2000). Simulating the consequences of a saccadic eye movement by fast motion of the retinal image during fixation yields a shift but no compression of apparent positions, which was taken as evidence that only the latter may be mediated by a corollary discharge associated with the oculomotor command (Morrone et al., 1997). However, a complete dissociation between real and simulated saccade conditions has been challenged by recent studies that found more perceptual compression around the apparent motion of visual stimuli under steady visual fixation than previously expected (Brenner et al., 2006; Ostendorf et al., 2006; Shim and Cavanagh, 2006; Watanabe and Yokoi, 2006). The latter finding may suggest that extraretinal signals associated with attention shifts or preparatory oculomotor activity contribute to perisaccadic perceptual compression.

The at least partial dissociation points toward a differential contribution of visual and extraretinal signals to shift and compression components of perisaccadic mislocalization. Furthermore, it may suggest that the brain relies on more than one extraretinal signal for perisaccadic localization, with these extraretinal signals contributing differentially to the two components of perisaccadic mislocalization (Bockisch and Miller, 1999; Kaiser and Lappe, 2004; Awater and Lappe, 2006): An anticipatory remapping of visual space starting before saccade onset may correspond to a presaccadic shift of apparent positions in saccade direction (Matin and Pearce, 1965; Honda, 1989; Dassonville et al., 1995; Cai et al., 1997). With visual references available, a presaccadic reafference of the saccade target (Moore, 1999) may induce an additional bias of apparent positions toward the target position (Honda, 1993; Morrone et al., 1997; Ross et al., 1997; Lappe et al., 2000; Kaiser and Lappe, 2004).

The observed correlation of the compression component with saccadic peak velocity allows for two major explanations. On the one hand, it may suggest that the level of perisaccadic perceptual compression varies with the strength of the corresponding saccadic motor command: saccadic peak velocities correlate with average firing rates of burst neurons recorded in the superior colliculi (Waitzman et al., 1991) and the pontomedullary reticular formation (Van Gisbergen et al., 1981). The superior colliculi and downstream structures provide an important source of reaf- ferent signals that are fed back to frontal and parietal oculomotor areas via thalamic relay nuclei (Buttner-Ennever and Henn, 1976; Sommer and Wurtz, 2002). It may thus be speculated that reafference of increased saccade-related neuronal activity in turn leads to a stronger bias of apparent positions toward the saccade target position (Kaiser and Lappe, 2004). This could provide a straightforward explanation why faster saccades lead to a stronger perisaccadic compression, implicating that at least the actual strength of perisaccadic compression is modulated by a reafferent extraretinal signal. Following this explanation, one should also expect an association of perisaccadic perceptual compression to saccadic reaction time (Stanford et al., 1996). Such a relationship might, however, have been masked by the actual task design with a fixed saccade direction across single blocks, enabling advance preparation of upcoming saccades (Dorris and Munoz, 1998): neurophysiological evidence indicates that phasic activity, like the burst of the actual saccadic command rather than slowly rising preparatory activity, is conveyed via thalamic relay nuclei to cortical structures (Sommer and Wurtz, 2002).

Alternatively, a purely visual account may principally explain a correlation of perisaccadic mislocalization with saccadic peak velocity as well: because of variable afferent delays (Schmolesky et al., 1998) and visible persistence (Coltheart, 1980), the retinotopic trace of a stimulus flashed around saccade onset may be erroneously integrated with an already moving or even postsaccadic visual reference frame (Schlag and Schlag-Rey, 2002; Pola, 2004; Ostendorf et al., 2006). In this context, a faster displacement of the retinal image per se could lead to larger mislocalization effects. However, velocity-dependent differences of such a putative integration process should manifest themselves primarily in the shift component of perceptual mislocalization, with faster displacements of the retinal image leading to a larger shift of apparent direction (MacKay, 1970). Higher retinal speed may not only lead to a less accurate alignment of a briefly presented stimulus with stable visual references and/or extraretinal signals but may also increase the spatiotemporal uncertainty of its representation. A less reliable representation may then be biased more strongly toward salient references (e.g., the saccade target), resulting in a stronger compression of apparent positions with higher retinal image speed (Brenner et al., 2006; Ostendorf et al., 2006). Following this hypothesis, not only the systematic bias but also the variability of perceptual localization should vary with saccadic peak velocity, which is not supported by our dataset: individual localization variability just after saccade onset (i.e., at the moment of maximal compression) did not correlate with mean saccadic peak velocity (supplemental Fig. $2 A, B$, available at www.jneurosci.org as supplemental material). Moreover, in accordance with a previous report (Bockisch and Miller, 1999), the localization scatter in our subjects increased already before saccade onset and remained elevated after completion of the eye movement, indicating that the intrasaccadic retinal smear per se did not cause the apparent localization uncertainty (supplemental Fig. 2C-E, available at www.jneurosci.org as supplemental material).

Visual and extraretinal explanations may, however, not necessarily be mutually exclusive. In fact, it seems likely that the processes involved in the phenomenon of perisaccadic compression will act on the mechanisms that already support perceptual localization under steady visual fixation. In this view, a saccaderelated extraretinal signal could work analogous to an attentional top-down signal that boosts the salience of a visual object by an increase of contrast and response gain at attended locations, respectively (Carrasco et al., 2004). An increased salience of the 
saccade target around saccade onset (Deubel and Schneider, 1996; Moore, 1999) may in turn lead to a larger bidirectional localization bias toward the target position. Indeed, the majority of collicular neurons projecting back to thalamic nuclei were classified as visuomotor neurons (Sommer and Wurtz, 2002), a class of neurons shown to be active during covert shift of visual attention as well (Ignashchenkova et al., 2004). The concept of the putative underlying extraretinal signal being commensurate with an attentional top-down signal (Awater and Lappe, 2006) could provide a unifying approach for similar mislocalization patterns around saccades and passive motion of the retinal image (Brenner et al., 2006; Ostendorf et al., 2006; Shim and Cavanagh, 2006; Watanabe and Yokoi, 2006). Here, the reported relationship between saccadic peak velocity and perisaccadic perceptual compression could serve as a powerful tool to further disentangle the respective contributions of visual, attentional, and saccaderelated extraretinal signals to perisaccadic mislocalization: an examination of perceptual compression in patients with pathologically slow saccades may help to further clarify the respective contributions of visual and extraretinal signals to perisaccadic localization and the cerebral mechanisms of visual stability across saccades.

\section{References}

Awater H, Lappe M (2006) Mislocalization of perceived saccade target position induced by perisaccadic visual stimulation. J Neurosci 26:12-20.

Awater H, Burr D, Lappe M, Morrone MC, Goldberg ME (2005) Effect of saccadic adaptation on localization of visual targets. J Neurophysiol 93:3605-3614.

Bahill AT, Clark MR, Stark L (1975) The main sequence, a tool for studying human eye movements. Math Biosci 24:191-204.

Bockisch CJ, Miller JM (1999) Different motor systems use similar damped eye position information. Vision Res 39:1025-1038.

Brenner E, van Beers RJ, Rotman G, Smeets JB (2006) The role of uncertainty in the systematic spatial mislocalization of moving objects. J Exp Psychol Hum Percept Perform 32:811-825.

Buttner-Ennever JA, Henn V (1976) An autoradiographic study of the pathways from the pontine reticular formation involved in horizontal eye movements. Brain Res 108:155-164.

Cai RH, Pouget A, Schlag-Rey M, Schlag J (1997) Perceived geometrical relationships affected by eye-movement signals. Nature 386:601-604

Carrasco M, Ling S, Read S (2004) Attention alters appearance. Nat Neurosci 7:308-313.

Coltheart M (1980) Iconic memory and visible persistence. Percept Psychophys 27:183-228.

Dassonville P, Schlag J, Schlag-Rey M (1995) The use of egocentric and exocentric location cues in saccadic programming. Vision Res 35:2191-2199.

Deubel H, Schneider WX (1996) Saccade target selection and object recognition: evidence for a common attentional mechanism. Vision Res 36:1827-1837.

Dorris MC, Munoz DP (1998) Saccadic probability influences motor preparation signals and time to saccade initiation. J Neurosci 18:7015-7026.

Honda H (1989) Perceptual localization of visual-stimuli flashed during saccades. Percept Psychophys 45:162-174.
Honda H (1993) Saccade-contingent displacement of the apparent position of visual-stimuli flashed on a dimly illuminated structured background. Vision Res 33:709-716.

Ignashchenkova A, Dicke PW, Haarmeier T, Thier P (2004) Neuronspecific contribution of the superior colliculus to overt and covert shifts of attention. Nat Neurosci 24:11236-11243.

Kaiser M, Lappe M (2004) Perisaccadic mislocalization orthogonal to saccade direction. Neuron 41:293-300.

Lappe M, Awater H, Krekelberg B (2000) Postsaccadic visual references generate presaccadic compression of space. Nature 403:892-895.

MacKay DM (1970) Mislocation of test flashes during saccadic image displacements. Nature 227:731-733.

Matin L, Pearce DG (1965) Visual perception of direction for stimuli during voluntary saccadic eye movements. Science 148:1485-1488.

Michels L, Lappe M (2004) Contrast dependency of sacadic compression and suppression. Vision Res 44:2327-2336.

Moore T (1999) Shape representations and visual guidance of saccadic eye movements. Science 285:1914-1917.

Morrone MC, Ross J, Burr DC (1997) Apparent position of visual targets during real and simulated saccadic eye movements. J Neurosci 17:7941-7953.

Morrone MC, Ma-Wyatt A, Ross J (2005) Seeing and ballistic pointing at perisaccadic targets. J Vis 5:741-754.

Ostendorf F, Fischer C, Gaymard B, Ploner CJ (2006) Perisaccadic mislocalization without saccadic eye movements. Neuroscience 137:737-745.

Pola J (2004) Models of the mechanism underlying perceived location of a perisaccadic flash. Vision Res 44:2799-2813.

Ross J, Morrone MC, Burr DC (1997) Compression of visual space before saccades. Nature 386:598-601.

Ross J, Morrone MC, Goldberg ME, Burr DC (2001) Changes in visual perception at the time of saccades. Trends Neurosci 24:113-121.

Schlag J, Schlag-Rey M (2002) Through the eye, slowly: delays and localization errors in the visual system. Nat Rev Neurosci 3:191-200.

Schmolesky MT, Wang Y, Hanes DP, Thompson KG, Leutgeb S, Schall JD, Leventhal AG (1998) Signal timing across the macaque visual system. J Neurophysiol 79:3272-3278.

Shim WM, Cavanagh P (2006) Bi-directional illusory position shifts toward the end point of apparent motion. Vision Res 46:3214-3222.

Sommer MA, Wurtz RH (2002) A pathway in primate brain for internal monitoring of movements. Science 296:1480-1482.

Sperry RW (1950) Neural basis of the spontaneous optokinetic response produced by visual inversion. J Comp Physiol Psychol 43:482-489.

Stanford TR, Freedman EG, Sparks DL (1996) Site and parameters of microstimulation: evidence for independent effects on the properties of saccades evoked from the primate superior colliculus. J Neurophysiol 76:3360-3381.

Van Gisbergen JA, Robinson DA, Gielen S (1981) A quantitative analysis of generation of saccadic eye movements by burst neurons. J Neurophysiol 45:417-442.

von Holst E, Mittelstaedt H (1950) Das Reafferenzprinzip (Wechselwirkung zwischen Zentralnervensystem und Peripherie). Naturwissenschaften 37:464-476.

Waitzman DM, Ma TP, Optican LM, Wurtz RH (1991) Superior colliculus neurons mediate the dynamic characteristics of saccades. J Neurophysiol 66:1716-1737.

Watanabe K, Yokoi K (2006) Object-based anisotropies in the flash-lag effect. Psychol Sci 17:728-735. 